December 1992,

PUPT-1369

\title{
THE THEORY OF TURBULENCE IN TWO DIMENSIONS
}

\author{
A.M. Polyakov \\ Physics Department, Princeton University, \\ Jadwin Hall, Princeton, NJ 08544-1000. \\ E-mail: polyakov@puhep1.princeton.edu
}

\begin{abstract}
The methods of conformal field theory are used to obtain the series of exact solutions of the fundamental equations of the theory of turbulence. The basic conjecture, proved to be self-consistent, is the conformal invariance of the inertial range. The resulting physical picture is different from the standard one, since the enstrophy transfer is catalyzed by the large scale motions. The theory gives some unambiguous predictions for the correlations in the inertial range.
\end{abstract}




\section{Introduction}

During the last thirty years we witnessed an amazing unification of physical ideas. Concepts and notions of the totally different regions of physics appeared to be almost isomorphic. Spontaneous symmetry breaking and renormalization group are the most notable examples.

In this paper I extend slightly this set of isomorphic ideas. Namely, I apply methods of conformal field theory ( CFT ) to the problem of two-dimensional turbulence. Short version of this paper has been published earlier [1].

The major puzzle in the theory of turbulence is the following. It is commonly believed, that at large Reynolds numbers we are dealing with the stationary statistical regime, describing velocity distribution. In other words, there should exist a time-independent probability $P=P\left[v_{\alpha}(x)\right]$ (where $v_{\alpha}(x)$ ) is the velocity). This probability should commute with the equations of motion, or:

$$
\int d x\left(\frac{\delta P}{\delta v_{\alpha}(x)}\right) \dot{v}_{\alpha}(x)=0
$$

(where it is understood that we express $\dot{v}_{\alpha}$ through $v_{\alpha}$ by the use of Navier - Stokes equations). At large Reynolds numbers viscosity can be neglected and hydrodynamics becomes a hamiltonian theory with the hamiltonian:

$$
H=\int d x \frac{1}{2} v_{\alpha}^{2}
$$

If so, the equation (1) means that $\mathrm{P}$ is the integral of motion for this system. Since it is believed that in general there are no non-trivial integrals for the system (2) (in twodimensions there are, but these are irrelevant for our discussion) we could erroneously conclude that:

$$
P=P(H)=\exp \{-\beta H\}
$$

(the last equality follows from the usual additivity assumption). This is the Gibbs distribution for the temperature $\beta^{-1}$ and it clearly does not describe the turbulent flow, in which we expect permanent energy flux.

The resolution of this puzzle is that in fact there are extra integrals of motion which form $P\left[v_{\alpha}\right]$. They are, however, highly non-local and non-polynomial. It would be difficult and unnecessary to write them down explicitly, because there still be a problem of averaging with respect to very complicated distributions. In what follows we will mostly use the analogue of the equation (1) applied directly to the correlation functions. Nevertheless we will briefly comment on the origin of the extra integral of motions for the systems with weak coupling. In this case they are just naive field-theoretic constants of motion formed of "in" and "out" operators. When expressed in terms of the Heisenberg fields they become non-local, and describe the distributions of weak turbulence. 
The central point of this paper is, however, quite different. We will consider the equations for the correlation functions:

$$
<\dot{v}_{\alpha_{1}}\left(x_{1}\right) v_{\alpha_{2}}\left(x_{2}\right) \cdots>+<v_{\alpha_{1}}\left(x_{1}\right) \dot{v}_{\alpha_{2}}\left(x_{2}\right) \cdots>+\cdots=0
$$

(where $\dot{v}_{\alpha}$ is expressed in terms of v by the use of equations of motion). These are standard equations (The Hopf equations [2] ) which express $\mathrm{N}$ - point functions in terms of the $\mathrm{N}$ +1 - point function. The existing procedures for dealing with these equations are based on some kind of closure hypothesis expressing, say the 4 - point function as a square of 2 - point function.

We take a different approach. Namely, we try to satisfy (4) exactly, by the use of conformal field theory, assuming that the developed turbulence in the inertial range possesses infinite conformal symmetry. There is no proof that this must necessarily the case, and that our conformal solutions are the only possible ones. What we have done is only a successful ansatz which solves exactly eqs (4).

\section{Chains of equations solved by conformal field the- ories.}

In this section I will sum up some basic properties of CFT, needed below as a representative example, I will use the most familiar case of the Ising model. The Ising model is a $\varphi^{4}$-field theory[3]. That means that if we treat $\varphi$ as a fluctuating variable, it satisfies the equation:

$$
\partial^{2} \varphi(x)+\mu \varphi(x)=g \varphi^{3}(x)
$$

implying the relations between $\mathrm{N}$ - point functions of $\varphi$ and $\mathrm{N}+3$ - point functions, known as Schwinger - Dyson equations. The constant $\mu_{0}$ must be fine - tuned in order to put the system to the critical point, or in order to allow (5) to have conformal invariant solutions. Now let us try to answer the basic question of this section : how CFT solves eqs (5)? In CFT we are dealing directly with the sets of correlation functions at the critical point. The basic assumptions [4] are that there exists a set of the so called primary operators $\left\{O_{k}(x)\right\}$ with anomalous dimensions $\left(\Delta_{k}, \bar{\Delta}_{k}\right)$ (referring to the rescaling of variables $z=x_{1}+i x_{2}$ and $\bar{z}=x_{1}-i x_{2}$. It is assumed that under arbitrary analytic transformation $z \Rightarrow f(z), O_{k}$ transform as:

$$
O_{k}(z, \bar{z}) \Longrightarrow\left(\frac{\partial f}{\partial z}\right)^{\Delta_{k}} \overline{\left(\frac{\partial f}{\partial z}\right)^{\Delta_{k}}} O_{k}(f, \bar{f})
$$

This transformation is generated by the analytic energy-momentum tensors $\mathrm{T}(\mathrm{z})$ and $\bar{T}(z)$ (related to the tensor $T_{\alpha \beta}(x)$ as $T, \bar{T}=T_{11}-T_{22} \pm 2 i T_{12}$ ). Conformal invariance implies that the trace of the energy-momentum tensor is zero. 
The basic result of [4] was that together with primary operators, CFT is bound to contain the so-called secondary or descendent operators. They come from the conformal deformations of the primary ones. In order to describe them it is convenient to introduce the Virasoro algebra:

$$
\begin{gathered}
L_{n}=\oint d z z^{n+1} T(z) \\
{\left[L_{n}, L_{m}\right]=(n-m) L_{n+m}+\frac{c}{12} n\left(n^{2}-1\right) \delta_{n+m, 0}}
\end{gathered}
$$

The secondary operators are given by: $O_{k}^{n_{1} \cdots n_{l}, m_{1} \cdots m_{n}}=L_{-n_{1}} \ldots L_{-n_{l}} \overline{L_{-m_{1}} \ldots L_{-m_{n}}} O_{k}$ The correlation functions of the secondaries are expressed by the differential relations through the correlators of the primaries [4].

The second basic feature of CFT is the operator product expansion. It says that

$$
O_{k}(z) O_{l}(0)=\sum f_{k l}^{m}(z \bar{z})^{\Delta_{m}-\Delta_{k}-\Delta_{l}} O_{m}(0)+\text { secondaries }
$$

The structure constants satisfy simple (in principal) consistency relation coming from the associativity of the algebra. Thus, the classification problem of CFT is reminiscent of the classification problem of the Lie algebras, but is vastly more complicated.

However, in [4] we have found a simplest set of CFT's, the so called minimal models. In these models the number of the primaries is finite (but the total number of operators is, of course, infinite). The Ising model is the minimal model of the type $(3,4)$ with only 3 primary operators. They are unit operator I , spin $\sigma$ and energy density $\varepsilon$. The fusion rules (9) in this case are:

$$
\begin{array}{r}
{[\sigma][\sigma]=[I]+[\varepsilon]} \\
{[\sigma][\varepsilon]=[\sigma]} \\
{[\varepsilon][\varepsilon]=[I]}
\end{array}
$$

The brackets in these symbolic formulas mean the "conformal class", namely the primary operator itself together with all it descendents.

Now we are coming to the central point. Let us identify the field $\varphi$ of the $\varphi^{4}$ theory with the field $\sigma$ of the conformal field theory. We have to examine the quantities $<\varphi^{3}(x) \varphi\left(y_{1}\right) \cdots \varphi\left(y_{n}\right)>$. However, the conformal field theory is applicable only when all points are well separated compared to the lattice spacing. The way out of this problem is to introduce the point - splitted definition of the $\varphi^{3}(x)$. Let us examine the object:

$$
\varlimsup_{a \rightarrow 0} \sigma\left(x+\frac{a}{2}\right) \sigma\left(x-\frac{a}{2}\right) \sigma(x) \equiv " \sigma^{3}(x) " \stackrel{\text { def }}{=} \Phi(x, a)
$$

The symbol $\overline{l i m}$ here means that we first of all average over directions of $a$ and then take $|a|$ to be much smaller then all other distances $\left|x-y_{i}\right|$ and $\left|y_{i}-y_{j}\right|$. Keeping it at first much larger than the lattice spacing, we can apply the fusion rules (9). Since

$$
[\sigma][\sigma][\sigma]=[\sigma]
$$


We obtain:

$$
\Phi(x, a) \approx|a|^{-4 \Delta_{\sigma}}\left[c_{1} \sigma(x)+c_{2} a^{2} \partial^{2} \sigma(x)+\ldots\right]
$$

Although this equation is correct only for $|a|>>l$ (where 1 is a lattice spacing), with any natural definition of the theory it can be extrapolated to $|a| \sim l$. The reason is that with any isotropic regularization at the distance we will get instead of (15):

$$
\Phi(x, a)=l^{-4 \Delta_{\sigma}} f\left(\frac{|a|}{l}\right) \sigma(x)+\ldots
$$

(where precise form of $f$ depends on the way we regulate the theory). As a result, if we take $a \lesssim l$ we still have the equation (15), and hence the basic field equation (5) is satisfied. All this means one simple thing -field equations of motion, when read from the right to the left are nothing but operator product expansions. This fact was well understood from the very beginning of the field theory for critical phenomena. Point-splitting and directional averaging over $a$ is also nothing new - already in quantum electro-dynamics it has been necessary to introduce the isotropic cut-off in the momentum space, which is equivalent to the procedure we discuss.

To summarize - in this section we have seen how the structure of the operator product expansion reflects equations, satisfied by the correlation functions.

\section{Solutions of the inviscid Hopf equations by the con- formal field theory.}

Let us turn now to the Hopf equations (4) and try to solve it in the spirit of the proceeding section. In two dimensions it is convenient to introduce vorticity $\omega$ and the stream function $\psi$ given by

$$
v_{\alpha}(x)=e_{\alpha \beta} \partial_{\beta} \psi \quad \omega(x)=e_{\alpha \beta} \partial_{\alpha} v_{\beta}=\partial^{2} \psi
$$

They satisfy Navier - Stokes equations:

$$
\dot{\omega}+e_{\alpha \beta} \partial_{\alpha} \psi \partial_{\beta} \partial^{2} \psi=\nu \partial^{2} \omega
$$

If the stirring force is present, it must be added to the RHS of (18). If we assume, that for the large Reynolds numbers there exist the inertial range of scales in which both viscosity and the stirring force are negligible, we can as a first step examine the inviscid Hopf equation:

$$
\begin{array}{r}
<\dot{\omega}\left(x_{1}\right) \omega\left(x_{2}\right) \cdots>+<\omega\left(x_{1}\right) \dot{\omega}\left(x_{2}\right) \cdots>+\cdots=0 \\
\dot{\omega}(x)=-" e_{\alpha \beta} \partial_{\alpha} \psi \partial_{\beta} \partial^{2} \psi "
\end{array}
$$

Here, just as in the previous section, we must be careful in defining the correlators at the coinciding points. The reason there have been tied to the existence of the lattice 
cut-off (where conformal theory fails). The reason here is analogous - we expect that in the momentum space only $\omega(k)$ with $\frac{1}{L}<|k|<\frac{1}{a}$ should satisfy (19). Here a is the ultraviolet cut-off proportional, as we see later, to some power of the viscosity $\nu$ while $\mathrm{L}$ is the infrared cut-off, dictated by the scale of the stirring force. The UV - cut - off in the momentum space means that we must use the point- splitted definition of the product in $(20)$

$$
" e_{\alpha \beta} \partial_{\alpha} \psi(x) \partial_{\beta} \partial^{2} \psi(x) "=\varlimsup_{a \rightarrow 0} e_{\alpha \beta} \partial_{\alpha} \psi(x+a) \partial_{\beta} \partial^{2} \psi(x)
$$

Let us assume now, that $\psi$ is a primary operator of some yet unknown conformal field theory and compute the RHS of (21) by the use of operator algebra. Suppose that we have the structure:

$$
\psi(x+a) \psi(x)=(a \bar{a})^{\Delta_{\phi}-2 \Delta_{\psi}}\{\phi(x)+\text { descendents }\}
$$

Here we introduced the operator $\phi$ which is the minimal dimension operator in the operator product (22). In unitary theories (as in the Ising model) it is a unit operator. However, only Gibbs states (from which "nothing disappears") are described by the unitary theories. In turbulence we have "flux states" from which conserved quantities leak away, and it is natural to expect that they will be described by the non-unitary theories. In this case most of operators have negative dimensions and $\phi$ is non-trivial. It is clear that when the operation (21) is applied to (22), the leading term in the expansion gives zero. This happens because the result must be pseudoscalar and we can't form it out of $\phi$ and its derivatives. Hence we have to consider subleading descendents in (22). They have the general structure:

$$
\begin{array}{r}
\text { descendents }=\sum c_{\{n\}\{m\}} L_{-n_{1}} \ldots L_{-n_{k}} \overline{L_{-m_{1}} \ldots L_{-m_{l}}} \phi(x) \\
(a \bar{a})^{\Delta_{\phi}-2 \Delta_{\psi}} a^{\sum n} \bar{a} \sum m
\end{array}
$$

Differentiation and directional averaging in (21) the leading term in (23):

$$
" e_{\alpha \beta} \partial_{\alpha} \psi(x) \partial_{\beta} \partial^{2} \psi(x) "=\operatorname{const}(a \bar{a})^{\Delta_{\phi}-2 \Delta_{\psi}}\left[L_{-2} \bar{L}_{-1}^{2}-\bar{L}_{-2} L_{-1}^{2}\right] \phi
$$

This one of the basic formulas of this work. The reason, why this descendent appeared in (24) is quite simple. The LHS of (24) is pseudoscalar and hence it changes sign under complex conjugation. The lowest descendent which has the same property is the one we find in (24). In deriving (24) we accounted for the UV - divergency, but disregarded the IR one. We will return to the infrared side of the problem below.

Let us assume for the moment that IR - divergencies are not present. If so, we derive a rather amazing conclusion from the eq.(24). Namely, there are two possibilities. The first one is $\Delta_{\phi} \leq 2 \Delta_{\psi}$ (negative:defect of dimensions"). In this case the only way to satisfy (19) would be to assume that the operator

$$
\Omega=\left(L_{2} \bar{L}_{-1}^{2}-\bar{L}_{-2} L_{-1}^{2}\right) \phi
$$


is either zero or is a symmetry of the underlying CFT. The latter statement just stresses the fact that the relation (19) implies that the infinitesimal variation of $\omega, \dot{\omega}=\Omega$ must not change the correlation functions, or as in the case of decaying turbulence rescale them. It is quite possible that non-trivial examples of such symmetries exist. However if we restrict ourselves with the simple minimal models, we will have to conclude that the RHS of (25) must be zero. This is easy to achieve if we require that the minimal dimension operator $\phi$ is degenerate on the level two. That means that it satisfies the equation [4]:

$$
\left(L_{-2}-\frac{3}{2\left(2 \Delta_{\phi}+1\right)} L_{-1}^{2}\right) \phi=0
$$

If so, $\Omega=0$. The simplest example, in which this relation is satisfied is the minimal model $(2,5)$ with $\Delta_{\phi}=\Delta_{\psi}=-\frac{1}{5}$

Even more surprising is the fact, that if $\Delta_{\phi}>2 \Delta_{\psi}$ then no extra restrictions are needed because as $a \rightarrow 0$ the RHS of (25) is zero, and the inviscid Hoft equation is satisfied. So, any CFT with the positive defect of dimensions solves the Hopf equations.

This is a "turbulent" counter part of the fact that there are continuosly many parasite solutions of the static Euler equations. Let us remember that if we look at the "laminary" solutions of

$$
e_{\alpha \beta} \partial_{\alpha} \psi \partial_{\beta} \partial^{2} \psi=0
$$

we find that if $\partial^{2} \psi=f(\psi)$ with arbitrary $f(\psi)$ then (27) is satisfied. It is well known, however, that matching with the viscous region by the boundary layer consideration removes this ambiguity. This analogy makes it clear that the crucial point for selecting correct solutions of the inviscid Hopf equations must be matching relations at the UV region of the cut-off momenta, where viscosity takes over. It is also clear, that since we are dealing with negative dimensions, we have to analyze the infrared diver gencies. We can say that in turbulence there are two boundary layers in the "momentum space", each requiring a separate theory. In the following sections we will discuss some partial results in this direction.

\section{The infrared problem}

The CFT's which are most interesting for the turbulence problem contain negative dimensions. That means that the formal correlation functions are positive powers of the distance. It is clear that we have to be very careful in the infrared region and treat it separately. Let us begin with the 2 - point function $<\psi(0) \psi(x)>$. When the dimension $\Delta_{\psi}<0$, CFT gives

$$
<\psi(0) \psi(x) \stackrel{\text { (conf) }}{>}=-|x|^{4\left|\Delta_{\psi}\right|}
$$

This is clearly unphysical. The correct prescription should take into account the fact, that

only momenta in the inertial range are described by CFT. So, we should expect that, if 
we take the Fourier transform of (28)

$$
<\psi(k) \psi(-k)>=\operatorname{const} \frac{1}{|k|^{2+4\left|\Delta_{\psi}\right|}}
$$

this result must be used only if $L^{-1}<|k|<a^{-1}$. We do not know the contribution from the $|k| \sim L^{-1}$. The only thing which can be said is that when returning to the $\mathrm{x}$ space it should give expressions, analytic in $x^{2}$. So, we have:

$$
<\psi(0) \psi(x)>\sim \int_{|k|} \gtrsim L^{-1} e^{i k x}|k|^{-2-4\left|\Delta_{\psi}\right|} \sim\left(c_{1} L^{4\left|\Delta_{\psi}\right|}+\ldots\right)-|x|^{4\left|\Delta_{\psi}\right|}
$$

with the first bracket representing the contribution of the infrared modes. When we turn to the multi - point functions, the situation is essentially the same. When we examine their momentum space form:

$$
G\left(k_{1} \cdots k_{N}\right)=<\psi\left(k_{1}\right) \cdots \psi\left(k_{N}\right)>\quad\left(\Sigma k_{j}=0\right)
$$

we will assume that the CFT formulas for this quantity work,provided that:

$$
L^{-1}<\left|k_{i}\right|<a^{-1}
$$

and

$$
L^{-1}<\left|k_{i 1}+\cdots+k_{i l}\right|<a^{-1}
$$

The (33) condition means that we have no small momenta transferred in any channel. Again, in the coordinate space all that means that the physical $\mathrm{N}$ - point function is equal to the conformal one plus the terms, analytic in some $\left(x_{i}-x_{j}\right)$. Notice, that if we understand the momentum integrals as analytically continued in $\left(\Delta_{\psi}\right)$ from the region, where they converge, we get precisely conformal answer in the $\mathrm{x}$ - space. In order to get the physical correlator within this analytic regularization, one has to add $\delta$ - functions in the $\mathrm{k}$ - space, representing the infrared modes. For instance, within the analytic prescription, we have instead of (29)

$$
<\psi(k) \psi(-k)>=\frac{\text { const }}{|k|^{2+4\left|\Delta_{\psi}\right|}}+a_{1} L^{4\left|\Delta_{\psi}\right|} \delta(k)+a_{2} L^{4\left|\Delta_{\psi}\right|-2} \frac{\partial^{2}}{\partial k^{2}} \delta(k)+\cdots
$$

When Fourier - transformed, (34) gives again (30).

So, the convenient way to summarize this situation is to say, that we have some sort of Bose - condensate in the momentum space, formed by the large scale motions. The physical correlators differ from the conformal ones by the condensate terms (we will call them $\delta$-terms later). The $\delta$ - terms occur whenever any sum of momenta in the correlator is equal to zero. In the coordinate space they are represented by the terms analytic in $\left(x_{i}-x_{j}\right)$. Occurrence of the $\delta$ - terms is not surprising, because we have strirring forces acting at $\mathrm{k}=0$. The main problem with them is that we do not know yet how to determine their form from the dynamics. The other related problem, which we will consider now, is 
whether they destroy our solution of the Hopf equations. The danger comes from the fact that in the sect. 3 we dealt with the purely conformal correlators, and this deri vation has to be reconsidered. In order to do that, let us rewrite our basic equation in the momentum space:

$$
<\dot{\omega}(q) \omega\left(f_{1}\right) \cdots \omega\left(f_{n}\right)>=-\int d^{2} k[k, q]\left((q-k)^{2}-k^{2}\right)<\psi(k) \psi(q-k) \omega\left(f_{1}\right) \cdots \omega\left(f_{n}\right)>
$$

Generally speaking, this integral has infrared divergency as $k \rightarrow 0$ (or $k \rightarrow q$ ). The results of the proceeding section guarantee that if we treat this IR divergency by the analytic regularization, the RHS of (35) will be zero, as before.

The problem now is to study what happens when we simply impose the IR cut off in (35). In order to do this, we need to know the behavior of the correlations functions when one of the momenta is much smaller then the others. Let us first evaluate the divergencies at $k \rightarrow 0$. This limit in the coordinate space is dominated by the large $\mathrm{R}$ limit in the Fourier transform:

$$
<\psi(k) \psi(q-k) \omega\left(f_{1}\right) \cdots \omega\left(f_{N}\right)>=\int d^{2} R e^{i k R}<\psi(R) \psi(0) \omega\left(f_{1}\right) \cdots \omega\left(f_{N}\right)>
$$

The large $\mathrm{R}$ behavior is governed by the fusion rule, where we replace all operators except $\psi(R)$ by $\psi(0)$. Using notations of [4], we have the formula:

$$
<\psi(R) \psi(0) \omega\left(f_{1}\right) \ldots>=\sum_{N}<0|\psi(R)| \Delta+N><\Delta+N\left|\psi(0) \omega\left(f_{1}\right) \ldots\right| 0>
$$

where the states $\mid \Delta+N>$ correspond to the secondary operators of the $\psi$. Using the fact that

$$
<0|\psi(R)| \Delta+N>\infty \frac{1}{R^{2 \Delta+N}}
$$

we obtain the following leading terms in the asymptotic expansion

$$
\begin{array}{r}
<\psi(k) \psi(q-k) \omega\left(f_{1}\right) \cdots \omega\left(f_{N}\right)>\approx \frac{c_{1}}{|k|^{6+\lambda}}<\Delta\left|\psi(q) \omega f_{1} \cdots \omega\left(f_{N}\right)\right| 0> \\
+c_{2}\left\{\frac{k_{+}}{|k|^{6+\lambda}}<\Delta \mid L_{1} \psi(q) \omega\left(f_{1}\right) \cdots \omega\left(f_{N}\right) 0>+c . c .\right\}+\cdots
\end{array}
$$

When we substitute (39) into (35), the first leading term drops out after integration over directions of $\mathrm{k}$ the second term does contribute. Simple computation gives:

$$
<\dot{\omega}(q) \omega\left(f_{1}\right) \cdots \omega\left(f_{n}\right)>=\mathrm{const}^{2+\lambda}\left\{q_{+}<\Delta\left|L_{1} \omega(q) \cdots \omega\left(f_{n}\right)\right|>- \text { c.c. }\right\}+O\left(L^{\lambda}\right)
$$

This leading term can be interpreted as fluctuating transport by the large scale eddies. Indeed, the relation (40) is equivalent to the operator equation:

$$
\dot{\omega}(x)=L^{2+\lambda} \hat{u}_{\alpha} \partial_{\alpha} \omega(x)
$$


Here we introduced a new $x$ - independent operators $\hat{u}_{ \pm}$by the rule:

$$
<0\left|\hat{u}_{+} \omega\left(x_{1}\right) \ldots \omega\left(x_{n}\right)\right| 0>=i<\Delta_{\psi}\left|L_{1} \omega\left(x_{1}\right) \ldots \omega\left(x_{n}\right)\right| 0>
$$

and $\hat{u}_{-}=\hat{u}_{+}^{*}$. It is clear, that although this infrared counterterm makes $\dot{\omega} \neq 0$, the Hopf equation is still satisfied, due to the translation invariance. As we go to the order of $L^{\lambda}$ counterterms we find more complicated forms. By the same technic it is possible to show, that the complete divergencies give the following equation:

$$
\dot{\omega}=\hat{u}_{\alpha} \partial_{\alpha} \omega+\hat{h}_{\alpha \beta} \partial_{\alpha} \partial_{\beta} \psi
$$

with the

$$
<0\left|\hat{h}_{++} \omega\left(x_{1}\right) \cdots \omega\left(x_{N}\right)\right| 0>\sim<\Delta\left|\left(L_{2}+\lambda L_{1}^{2}\right) \omega\left(x_{1}\right) \cdots \omega\left(x_{N}\right)\right| 0>
$$

(where $\hat{h}_{\alpha \beta^{-}}$is a traceless tensor). This contribution does not cancel in the Hopf equation. There are also other infrared counterterm with this property, occurring from the region in the (45) where: $\left|k+f_{i 1}+\cdots+f_{i l}\right| \rightarrow 0$ (for some choice of $\left\{f_{i}\right\}$ ). All that means that if we had defined the physical correlation functions by simply cutting of the momentum integrals in the Fourier transform, the Hopf equation would be destroyed. On the other hand, if we had defined all integrals by analytic regularization it would mean the identification of the physical and conformal correlators. In this case the Hopf equations is satisfied, but the positivity is broken. The way out of this problem is to find the $\delta$ terms which do not spoil the Hopf equation but restore the positivity. The most blatant violation of positivity is easily cured, if we postulate that any correlation function contains the constant part, coming from the zero modes:

$$
<O_{n 1}\left(x_{1}\right) O_{n 2}\left(x_{2}\right) \cdots O_{n N}\left(x_{N}\right)>^{p h y s}=c_{n 1} \cdots n_{N}\left(L^{-2 \sum_{j} \Delta_{n j}}\right)+<O_{n 1} \cdots O_{n N}>^{\text {conf }}
$$

(where $\mathrm{C}$ - are some constants). This type of $\delta$ - term does not contribute to the Hopf equations at non - zero momenta, and at the same time prevails over the negative conformal contributions, since $\left|x_{i}-x_{j}\right| \ll L$. That doesn't mean that there could be no other IR counterterms. In fact it is possible to show that there are many types of the $\delta$ - terms, consistent with the Hopf equations at $q_{i} \neq 0$. In order to determine them we need to include the stirring force terms (which arise at $q_{i}=0$ ) and to use the matching relations. This is the problem for the future work. At present it is not quite clear to what extent the $\delta$ - terms are universal i.e. independent of the large scale structures.

There is another related issue which we should discuss in this section. It is the question of the vacuum expectation values of different operators. When defining our conformal contributions, we have implicitly assumed that all these expectation values (VEV) are zero. In general, however we have:

$$
<O_{n}(x)>=C_{n} \cdot L^{-2 \Delta_{n}}
$$

\footnotetext{
${ }^{1}$ I am grateful to A. Zamolodchicov for drawing my attention to it
} 
If $\Delta_{n}<0$, and $c_{n} \neq 0$ these VEV considerably modify correlation functions, as was noticed by Al. Zamolodchikov[5 ]. The reason for that is simple. From the OPE we get:

$$
<\psi(r) \psi(0)>=r^{-4 \Delta \psi}<I>+r^{2\left(\Delta_{\phi}-2 \Delta_{\psi}\right)}<\phi>+\cdots=r^{4\left|\Delta_{\psi}\right|}\left\{1+<\phi>\cdot\left(\frac{L}{r}\right)^{2\left|\Delta_{\phi}\right|}+\cdots\right\}
$$

The second term in (48) is the dominating one. The question, whether VEV are non-zero is determined by the infrared boundary conditions. Our assumption that $c_{n}=0$ is not fundamental. Actually our consideration of the Hopf equations remains intact even if $c_{n} \neq 0$. The only thing which becomes more complicated is the infrared divergency. Also, the UV - matching of the next section requires modified consideration.

The question of the $c_{n}$ values belongs to the same category of the IR problems which we left for the future work. At present the situation resembles quantum chromodynamics, where we have not solved the IR problem, but can, nevertheless, test its small distance behaviour.

Let us summarize. The true physical correlators can be obtained from the conformal ones either by defining the momentum space integrals in the sense of analytic regularization or in the usual sense, with the IR cut - off. In both cases it is necessary to add to these expressions the $\delta$-terms, containing $\delta$ functions of certain external or transferred momenta. These terms in the coordinate space contain the analytic dependence on certain distances. Precise form of the $\delta$ - terms requires for its definition the matching with the stirring force - the task not accomplished in this paper.

\section{The Flux States}

In the above considerations we accounted for viscosity in a somewhat symbolic sense. Namely, we have used the inviscid Hopf equations, but assumed that at the very high momenta our power-like correlation functions start to drop rapidly because of the viscosity, which was assumed as the origin of the ultra- violet cut-off.

It is clear that the precise nature of this cut-off must be important. For instance, we can imagine an "elastic" cut-off which preserves the hamiltonian structure and the "inelastic" one which introduces dissipation. It is clear that they should correspond to different physics.

In this section we' ll try to impose the dissipation condition on the theory. The basic idea is roughly the same as in the classical Kolmogorov treatment of turbulence. Namely, we will assume (self-consistently) that integrals of motion are dissipated at the UV cut-off, and in the inertial range of momenta they are just transferred. It implies that the flux in the momentum space of conserved integrals must be constant. One can say that while Gibbs distributions are uniform on the surfaces of fixed values of conserved quantities, the turbulent distributions are located on the surfaces of the constant fluxes 
of the corresponding quantities.

The constant flux in the momentum space is also a familiar object in the other part of physics. It is responsible for the anomalies in the quantum field theory. When one considers, for example, massless fermions in the electromagnetic field the chirality (the number of left minus the number of right Dirac particles), is not conserved due to axial anomaly. This happens because the ultra-violet regularization breaks conservation of the axial current. After the chirality is injected at the UV cut-off, it propagates in the momentum space to the physical region. There is, therefore, a clear and useful analogy with what we are discussing.

It has been noticed by Kraichnan [6], that in two dimensions the most important flux is that of enstrophy (we will comment on that later). Let us derive the constraint on the theory which follows from the enstrophy conservation. Consider first the enstrophy contained in the modes with definite momentum:

$$
h(\vec{k})=<\omega(\vec{k}) \omega(\overrightarrow{-k})>
$$

Without viscosity and external force, $h(\vec{k})$ would satisfy the continuity equation in the momentum space. When these factors are accounted for, the equation becomes:

$$
\dot{h}(k)+\frac{\partial}{\partial k_{i}} J_{i}^{(h)}=\nu k^{2} h(k)+\Phi(k)
$$

Here $\Phi(\vec{k})$ is a contribution of the external forces which is non-zero only for $|\vec{k}| \sim \frac{1}{L}$. Let us notice also, that in isotropic turbulence the only non-zero component of $\vec{J}^{(h)}$ is the radial one $J^{(h)}(k)$. Let us now chose the momentum q lying in the inertial range and integrate over $|\vec{k}|>|\vec{q}|$. Since in the steady state $\dot{h}(k)=0$ we get

$$
-J^{(h)}(q)=\nu \int_{|k|>|q|} k^{2} h(k) d^{2} k
$$

Now comes an important point - suppose that the RHS of (51) is UV- divergent and defined by $|k| \sim \frac{1}{a}$. Then the q-dependence of RHS can be neglected. As a result, we obtain:

$$
J^{(h)}(q) \approx-\nu \int k^{2} h(k) d^{2} k=\mathrm{const}
$$

for $\frac{1}{L} \ll|q| \ll \frac{1}{a}$. The meaning of this constant flux condition is transparent. It just says that enstrophy is dissipated only at $|q| \sim \frac{1}{a}$ and hence, the conservation law implies that its flux must be constant in the inertial range. This is one of matching conditions we must impose on the inviscid solutions. If we express $\omega \dot{(q)}$ by means of eq. (35) we get:

$$
J^{(h)}(q)=-\int_{|k|>q}<\dot{\omega(k)} \omega(-k)>=\int_{|k|<q}<\dot{\omega}(k) \omega(-k)>
$$

(we used here the fact, that (35) conserves total enstrophy). We see from here that all $\vec{k}-s$ from the inertial range give zero contribution. At the same time, using our general conjecture concerning the infrared terms (46) we obtain:

$$
J^{(h)}(q) \propto \frac{1}{L^{\Delta_{\dot{\omega}}+\Delta_{\omega}}}
$$


The right hand side of (51) is independent of L and thus we obtain the matching condition:

$$
\Delta_{\omega}+\Delta_{\dot{\omega}}=0
$$

If we had used the first term in ( 52$)$, where $k \sim \frac{1}{L}$ are absent, the IR contribution would come from the integral (35) for $\dot{\omega}$, yielding the same result. If we recall, that: $\Delta_{\omega}=\Delta_{\psi}+1$ and $\Delta_{\dot{\omega}}=\Delta_{\phi}+2$ we get a following condition for the enstrophy flux state:

$$
\begin{array}{r}
{[\psi][\psi]=[\phi]+\ldots} \\
\Delta_{\psi}+\Delta_{\phi}+3=0
\end{array}
$$

This result heavily depends on our conjectures about IR - contributions, namely on the fact that $\langle\omega \dot{\omega}\rangle$ infrared part is non-zero. Had we considered the energy flux state, the formula ( 55) would be replaced by:

$$
\Delta_{\psi}+\Delta_{\phi}+2=0
$$

Apart from the energy and the enstrophy there are also higher conserved integrals of the type:

$$
I_{n}=\int \omega^{n}(x) d^{2} x
$$

Before commenting on these integrals, let us explain why the formulas (56) and (57) do not contradict each other. (After all, energy and enstrophy are both conserved in the inviscid system). The apparent contradiction is removed by the fact that in the enstrophy flux state the integral for the energy dissipation, analogous to (50) is given by:

$$
J^{(\varepsilon)}(q)=\nu \int_{|k|>q} d^{2} k<\omega(k) \omega(-k)>
$$

Contrary to (50), (58) is not UV - divergent and the dissipation is scale - dependent in the inertial range.

In this case we can't use (51) anymore. No obvious matching condition arise in this case, since (58) tells us that as $\nu \rightarrow 0$ the energy flux tends to zero, while the enstrophy flux persists. This is essentially the picture, advocated by Kraichnan [6].

As we turn to the integrals $I_{n}$ we have to decide, whether they have nonzero flux and whether this gives us any new information. We are unable at this point to give complete analyses of this question and will present some non-rigorous estimates, leaving complete resolution for the future work. We have:

$$
\dot{I}_{n}=\nu \int<\nabla^{2} \omega(x) \omega^{n-1}(x)>d^{2} x
$$

The main problem is how to regularize this expression. We must use OPE in order to define $\omega^{n-1}$.If $\mathrm{n}$ is even, which is imposed by parity conservation, we get

$$
\omega^{n-1}(x) \infty \Psi(x)+\cdots
$$


and as a result:

$$
\dot{I}_{n} \propto \int<\Delta \omega \psi>\propto J^{(\varepsilon)}
$$

We picked up $\psi$ operator only in(61) since due to orthogonality of primary operators, all others will not contribute. This implies that the $I_{n^{-}}$transfer is zero in the inviscid limit and there are no extra constraints on the theory coming from it (apart, may be from some inequalities). However, this line of arguments must be considered in more details (with careful point - splitted definition of all parties involved, before the last conclusion could be trusted). In particular,the other groupings of $\omega$-s may involve some other operators. Much work here is to be done.

An interesting related question is that of the inverse cascades of higher integrals. [ Strong infrared divergency in their dissipation makes them very similar to the energy and opens this possibility for $I_{n}$. Whether this is the case is an unsolved question. Unfortunately, any kind of the naive pseudophysical arguments is misleading here.

To conclude this section, let us discuss the physical picture of the flux state. It is not exactly the Kolmogorov's one. We have constant flux of conserved quantity through the inertial range. In the Kolmogorov's mechanism the transfer occurs by the interaction of three modes with all wave vectors lying in the inertial range. Such transfer is absent in our picture. Instead we have the triad interaction in which one of the modes is infrared. We can say that the infrared (large scale) modes serve as catalyzers for the flux. This is an important qualitative prediction of our theory, which can be tested.

\section{Possible spectra of conformal turbulence.}

Let us discuss solutions of conformal turbulence with constant enstrophy flux. The analyses of the previous sections indicates that we must start from the CFT, satisfying certain constrains which follow from the Hopf equation and constant flux condition. The first constraint tells us that if we have the fusion rule:

$$
[\psi][\psi]=[\phi]+\cdots
$$

where $\phi$ is a minimal dimension operator in the product (62), then it must be either $\Delta_{\phi}>2 \Delta_{\psi}$ or $\phi$ - operator must be degenerate at the level two.

The second constraint depends on the assumption about vacuum expectation values. The simplest version of the theory, when these values are zero gives:

$$
\Delta_{\phi}+\Delta_{\psi}=-3
$$

More options arise if we assume that if $\left\langle O_{n}\right\rangle \neq 0$ In this case the correlation functions $\omega \omega$ and $\dot{\omega} \omega$ are determined not by the unit operator but by the minimal dimension

\footnotetext{
${ }^{2}$ I acknowledge useful discussion of this point with G. Falcovich and A. Hanany
} 
operators. As a result, if we look at OPE, we find:

$$
\begin{aligned}
<\omega(z) \omega(0)>\propto(\bar{z} z)^{\Delta_{\phi}-2 \Delta_{\omega}} & <\phi> \\
\Delta_{\omega}= & \Delta_{\psi}+1
\end{aligned}
$$

and

$$
<\dot{\omega} \omega>\propto(\bar{z} z)^{-\left(\Delta_{\omega}+\Delta_{\dot{\omega}}\right)}(\bar{z} z)^{\Delta_{\chi}}
$$

where $\chi$ is a minimal dimension operator in the product $[\psi][\psi][\psi]$. So, in this case the constant flux condition is replaced by:

$$
\Delta_{\phi}+\Delta_{\psi}-\Delta_{\chi}=-3
$$

while the energy spectrum is given by:

$$
E(k) \sim k^{4 \Delta_{\psi}-2 \Delta_{\phi}+1}
$$

One can also consider intermediate possibilities when some of the operators have vacuum averages and some do not.

At present we do not have clear idea how the choice should be made. It may depend on the external conditions and the way turbulence is generated.

Below we will examine a simplest possible model, the "minimal" minimal model. By that we mean the theory which satisfies our requirements and has the smallest number of primaries. If we associate each primary operator with the certain type of motion then it is indeed the simplest (but by no means the only) type of turbulence. Let us look at the minimal models of the type $(2,2 \mathrm{~N}+1)$, and assume, that vacuum expectation values are absent. In this models we have the set of $\mathrm{N}$ primary operators $\left[\psi_{s}\right]$, with $s=1, \cdots N$. The fusion rules are:

$$
\begin{array}{r}
{\left[\psi_{s_{1}}\right]\left[\psi_{s_{2}}\right]=\left[\psi_{s_{3}}\right]+\left[\psi_{s_{3}-2}\right]+\ldots} \\
s_{3}=\min \left(s_{1}+s_{2}-1,2 N+1-\left(s_{1}+s_{2}-1\right)\right)
\end{array}
$$

The dimensions are given by:

$$
\Delta_{s}=-\frac{(2 N-s)(s-1)}{2(2 N+1)}
$$

If we identify the stream function $\psi$ with the primary field $\psi_{S}$ with some $\mathrm{S}$, then the flux condition gives:

$$
\Delta_{s}+\Delta_{2 s-1}=-3
$$

(if $2(2 s-1)<2 N+1)$. This diophantine equation has unique solution, $\mathrm{s}=4 ; \mathrm{N}=10$. Thus, we conclude that the minimal turbulence is described by the $(2,21)$ minimal model. Anomalous dimensions in this case are

$$
\begin{gathered}
\Delta_{\psi}=\Delta_{4}=-\frac{8}{7} \\
\Delta_{\phi}=\Delta_{7}=-\frac{13}{7}
\end{gathered}
$$


As a result we obtain in this case the energy spectrum

$$
E(k) \infty k^{-\frac{25}{7}}
$$

It seems to be consistent with observations. [7]

An interesting question if this model is parity conservation. There is no a priori reason why we should exclude parity breaking solutions from the consideration. Let us look what is the situation in our model. The stream function $\psi$ is a pseudoscalar. That means that if parity is conserved, the 3 - point function must contain vector products. But in CFT this is impossible: all 3-point functions are simple powers of distances.[ Hence, in conformal turbulence the necessary condition for parity conservation is:

$$
<\psi \psi \psi>=0
$$

This is indeed the case, since:

$$
\left[\psi_{4}\right]\left[\psi_{4}\right]=\left[\psi_{7}\right]+\left[\psi_{5}\right]+\left[\psi_{3}\right]+\left[\psi_{1}\right]
$$

and there is no $\left[\psi_{4}\right]$ in the RHS. As we look at higher correlations the situation is less clear. Indeed, the 5 - point functions of $\psi$ are still zero (by the similar arguments) while the 7 -point functions are non-zero. If parity is conserved, they must contain vector products. It is not obvious, whether this can be done, or, in other words, whether we can combine conformal blocks in the 7 - point function in a antisymmetric (under reflections) way. This we leave for the future analyses, noticing only, that if we consider the standard symmetric combinations of the conformal blocks, we get a very peculiar picture in this model. namely, the parity violation, if present at all, is well hidden - one must go to the seven - point functions in order to notice it!

Let us point out that parity is automaticaly conserved in the non-minimal models. In this case there exists infinite number of the degenerate operators, forming closed algebra preserving parity [4].

\section{Conclusion.}

There are infinitely many other solutions of the flux conditions, the fixed points with larger number of structures. They have been considered in [8]. It is hard to say, whether all matching conditions have been exploited and whether we indeed have infinite number of discrete fixed points. If so, the turbulent fluid has infinite discrete regimes and must be able to jump from one regime to the other, generating new quasistable structures. we are not in a position yet to prove this conjecture, but it is certainly within the scope of our methods.

\footnotetext{
${ }^{3}$ Some time ago A. Migdal conjectured that in $3 \mathrm{~d}$ turbulence $\mathrm{T}$ - invariance forbids the 3 -point functions. Whether it is true is an interesting open question. In our case the only thing which can be proved is the combined PT-invariance .
} 
As far as observations are concerned, the best way to check conformal invariance is to study momentum representation of the correlation functions when all the momenta and all their partial sums belong to the inertial range. In this way we avoid the infrared region which is scarcely understood.

The situation is analogous to that in quantum chromodynamics - there we know dynamics at high momenta and quite ignorant in the infrared. Nevertheless, QCD has been tested.

The theory of conformal turbulence gives explicit and unambiguous predictions concerning these type of correlators. They are expressed in terms of hypergeometric functions, and, more importantly have specific "fusion rule" structure when one momentum is getting much larger than the others. The most direct way to test the theory is to check this fusion property. For example, one takes the 4 - point function and reduce it to the 3 point function by taking one large momentum. This 3 - point function in the momentum space is a simple hypergeometric function, which becomes just a product of two powers if one does the fusion procedure again. This test is especially important, since we are not certain which solution of conformal turbulence is realized in a given experiment. Since the structure of fusion rules define the theory the test is capable to answer this question. On the other hand, may be the most interesting part of the theory is the infrared one, still hidden from us. With further work it might become possible to get equations, governing the behavior of the vacuum expectation values of $\left\langle O_{n}\right\rangle$ in space and time. This is the problem analogous to the one of equation of state in critical phenomena. In any case, the theory presented here is but a first step in the long future investigations.

\section{Acknowledgements}

I am grateful to A.Migdal, E.Siggia, V.Yakhot and A.Zamolodchikov for many important remarks on this work. I also thank D. Makogonenko for her help in preparation of this

article. This work was partially supported by the National Science Foundation under contract PHYS-90-21984. 


\section{REFERENCES}

[1] A.Polyakov Preprint PUPT-1341 (1992) (Princeton)

[2] A.Monin A.Yaglom, "Statistical Fluid Mechanics" MIT Press, Cambridge (1975)

[3] J.Kogut, K.Wilson Phys. Rep., 12C, 2, (1974)

[4] A. Belavin, A. Polyakov, A. Zamolodchicov, Nucl. Phys. B241, 33, (1984)

[5] Al. Zamolodchicov, Nucl. Phys. B358, 497, (1991)

[6] R. Kraichnan, Phys. of Fluids 10, 1417, (1967)

[7] B. Legras, P. Santangelo, R. Benzi, Europhys. Lett. 5 (1) 37, (1988)

[8] D. Lowe Preprint PUPT-1362 (1992) (Princeton)

G. Falcovich, A. Hanany, Weizman Institute Preprint (1992)

V. Pokrovsky, private communication 